\title{
A THEORY OF PROPER SHAPE FOR LOCALLY COMPACT METRIC SPACES
}

\author{
BY B. J. BALL AND R. B. SHER ${ }^{1}$
}

Communicated by R. D. Anderson, March 19, 1973

The notion of topological shape, first introduced by $\mathrm{K}$. Borsuk $([1],[2])$ for compact metric spaces, has been extended to larger classes of spaces in a number of ways $([3],[4],[10],[6],[8],[11])$. Not all of these extensions agree, but all retain the property of the original formulation that any two spaces of the same homotopy type have the same shape. Any extension to noncompact spaces which retains this property, however, must necessarily sacrifice much of the geometric flavor of the original definition, since homotopy type is notoriously ungeometric in the noncompact case. For noncompact spaces which are locally nice (e.g., locally compact ANR's), proper homotopy type gives a much more geometric classification. Since the proper homotopy type of a compactum (in the class of compacta) is the same as its homotopy type, Borsuk's original concept of shape could, perhaps, more appropriately be considered a generalization of proper homotopy type than of homotopy type, and extended to noncompact spaces so as to retain this property.

Of several natural extensions which apply to classes of spaces including noncompact ones and which generalize proper homotopy type, we have chosen to present one which, although limited to locally compact metrizable spaces, seems to emphasize geometric properties to a greater extent than do some alternatives which apply to larger classes of spaces. Moreover, this particular treatment very closely parallels Borsuk's original development, and in many instances virtually the same proofs apply. A detailed version, along with proofs of the results claimed here, will be published at a later date.

In order to avoid difficulties arising from the fact that closed sets in noncompact spaces may not have countable neighborhood bases, we substitute "fundamental nets" $\left\{f_{\lambda} \mid \lambda \in \Lambda\right\}, \Lambda$ a directed set, for the fundamental sequences $\left\{f_{k} \mid k=1,2, \ldots\right\}$ of Borsuk. And in order that our extension should generalize proper homotopy type rather than homotopy type, we require that all homotopies involved in the definitions should

AMS (MOS) subject classifications (1970). Primary 55D99; Secondary 54E45, 54D35, $54 \mathrm{~F} 40$.

Key words and phrases. Shape theory, proper homotopy theory, proper shape theory, ANR, one-point compactification, Freudenthal compactification.

1 The second named author was supported in part by NSF Grant GP-29585A \#1. 
be proper (on closed neighborhoods). Since every separable, locally compact, metrizable space can be embedded as a closed subset of the Hilbert cube minus a point, it turns out that we need consider, for the separable case, only the closed subsets of this particular space.

Specifically, if $K=H-\{\omega\}$, where $H$ is the Hilbert cube and $\omega \in H$, then a proper fundamental net from $X$ to $Y$ in $K$ is an ordered triple $(f, X, Y)$, where $X$ and $Y$ are closed subsets of $K$ and $f=\left\{f_{\lambda} \mid \lambda \in \Lambda\right\}$ is a family of maps $f_{\lambda}: K \rightarrow K$, indexed by a directed set $\Lambda$, such that:

$\left.{ }^{*}\right)$ For every closed neighborhood $V$ of $Y$ (in $\left.K\right)$, there exist a closed neighborhood $U$ of $X$ (in $K$ ) and an index $\lambda_{0} \in \Lambda$ such that for all indices $\lambda \geqq \lambda_{0}, f_{\lambda}\left|U \cong_{p} f_{\lambda_{0}}\right| U$ in $V$ (where $\cong_{p}$ denotes proper homotopy).

Two proper fundamental nets $f=\left\{f_{\lambda} \mid \lambda \in \Lambda\right\}$ and $g=\left\{g_{\delta} \mid \delta \in \Delta\right\}$ from $X$ to $Y$ in $K$ are said to be properly homotopic, denoted by $\boldsymbol{f} \cong_{p} g$, provided that:

$\left.{ }^{* *}\right)$ For every closed neighborhood $V$ of $Y$ (in $\left.K\right)$ there exist a closed neighborhood $U$ of $X$ (in $K$ ) and indices $\lambda_{0} \in \Lambda, \delta_{0} \in \Delta$ such that for $\lambda \geqq \lambda_{0}$ and $\delta \geqq \delta_{0}, f_{\lambda}\left|U \cong_{p} g_{\delta}\right| U$ in $V$.

The identity proper fundamental net from $X$ to $X$ in $K(X$ a closed subset of $K)$ is the triple $\boldsymbol{i}_{X}=(\boldsymbol{i}, X, X)$, where $\boldsymbol{i}$ is the degenerate net consisting only of the identity map $i_{K}: K \rightarrow K$.

The composition of the proper fundamental nets $f=\left\{f_{\lambda} \mid \lambda \in \Lambda\right\}$ from $X$ to $Y$ and $g=\left\{g_{\delta} \mid \delta \in \Delta\right\}$ from $Y$ to $Z$ is the proper fundamental net $\boldsymbol{g f}=\left\{g_{\delta} f_{\lambda} \mid(\lambda, \delta) \in \Lambda \times \Delta\right\}$ from $X$ to $Z$, where $\Lambda \times \Delta$ is directed by the relation $(\lambda, \delta) \geqq\left(\lambda_{0}, \delta_{0}\right)$ if and only if $\lambda \geqq \lambda_{0}$ and $\delta \geqq \delta_{0}$.

Two closed subsets $X$ and $Y$ of $K$ are then said to be properly fundamentally equivalent if there exist proper fundamental nets $f: X \rightarrow Y$ and $g: Y \rightarrow X$ such that $g f \cong_{p} i_{X}$ and $f g \cong_{p} i_{Y}$.

It can be shown that the proper fundamental equivalence of two closed subsets of $K$ is independent of their embeddings as closed subsets of $K$; thus we may define the statement " $X$ and $Y$ have the same proper shape" (which will be denoted by $\operatorname{Sh}_{p} X=\operatorname{Sh}_{p} Y$ ) for locally compact, separable, metrizable spaces $X$ and $Y$ to mean that there exist closed subsets $X^{\prime}$ and $Y^{\prime}$ of $K$ homeomorphic to $X$ and $Y$, respectively, such that $X^{\prime}$ and $Y^{\prime}$ are properly fundamentally equivalent in $K$. This induces an equivalence relation on the class of locally compact, separable, metrizable spaces, and, for such spaces,

(i) if $X \cong_{p} Y$, then $\operatorname{Sh}_{p} X=\operatorname{Sh}_{p} Y$, and

(ii) if $X, Y \in \mathrm{ANR}$ and $\operatorname{Sh}_{p} X=\operatorname{Sh}_{p} Y$, then $X \cong_{p} Y$.

It easily follows that two compact metric spaces have the same proper shape if and only if they have the same shape in Borsuk's original sense; in addition, no compact metric space can have the same proper shape as any noncompact (separable, locally compact) space. Moreover, if $X$ and 
$Y$ are separable, locally compact, metrizable spaces and $X^{*}=X \cup\{\infty\}$ and $Y^{*}=Y \cup\{\infty\}$ are the one-point compactifications of $X$ and $Y$, respectively, then the pointed spaces $\left(X^{*}, \infty\right)$ and $\left(Y^{*}, \infty\right)$ have the same pointed shape in the sense of Borsuk [1]; a stronger result holds, in fact, in that the point $\infty$ can be kept fixed at all stages of the homotopies involved. A similar result holds for the (Freudenthal [7]) endpoint compactifications $F X$ and $F Y$ of $X$ and $Y$ in case these are metrizable; here we have the result that if $\operatorname{Sh}_{p} X=\operatorname{Sh}_{p} Y$, then $(F X, E X)$ and $(F Y, E Y)$ have the same shape as pairs in the sense of [1], and therefore [9] in the sense of [10], where $E X$ and $E Y$ denote the endpoint sets $F X-X$ and $F Y-Y$, respectively. Again, a stronger result is available, in that $E X$ and $E Y$ can be kept pointwise fixed throughout all the homotopies involved. In particular, it follows that the number of ends (indeed, the homeomorphism class of the set of endpoints) of a connected manifold or other appropriate space is a proper shape invariant.

Another corollary, similar to a well-known result of Chapman [5], is that if $X$ and $Y$ are closed $Z$-sets in $K$ and $\operatorname{Sh}_{p} X=\operatorname{Sh}_{p} Y$, then $K-X \approx$ $K-Y$. The converse is, of course, false.

For nonseparable spaces, we use the fact that every locally compact metrizable space may be partitioned into (disjoint, open and closed) separable subspaces, and say that two locally compact metrizable spaces $X$ and $Y$ have the same proper shape if there exist partitions $X=\bigcup_{\alpha \in A} X_{\alpha}$ and $Y=\bigcup_{\alpha \in A} Y_{\alpha}$ of $X$ and $Y$ into separable subspaces such that $\operatorname{Sh}_{p} X_{\alpha}=\operatorname{Sh}_{p} Y_{\alpha}$, in the sense previously defined, for each $\alpha \in A$. It can be shown that this establishes an equivalence relation on the class of all locally compact metrizable spaces (transitivity requires some argument), and we have the result that two locally compact metrizable spaces which are of the same proper homotopy type have the same proper shape, and conversely for locally compact ANR's.

\section{REFERENCES}

1. K. Borsuk, Concerning homotopy properties of compacta, Fund. Math. 62 (1968), 223-254. MR 37 \#4811.

2. - Concerning the notion of the shape of compacta, Proc. Internat. Sympos. Topology and its Applications (Herceg-Novi, 1968), Savez Društava Mat. Fiz. i Astronom., Belgrade, 1969, pp. 98-104. MR 43 \#1138.

3. - On the concept of shape for metrizable spaces, Bull. Acad. Polon. Sci. Sér. Sci. Math. Astronom. Phys. 18 (1970), 127-132. MR 42 \#8443.

4. - Some remarks on the theory of shape in arbitrary metrizable spaces, Proc. Third Prague Sympos. on General Topology, (1971), Academic Press, New York, 1973.

5. T. A. Chapman, Some applications of infinite dimensional manifolds to the theory of shape, Fund. Math. 76 (1972), 181-193.

6. Ralph H. Fox, On shape, Fund. Math. 74 (1972), 47-71. MR 45 \#5973. 
7. H. Freudenthal, Neuaufbauen der Endentheorie, Ann. of Math. (2) 43 (1942), 261-279. MR 3, 315.

8. Sibe Mardešić, Shapes for topological spaces, Gen. Topology and Appl. (to appear).

9. - On Borsuk's shape theory for compact pairs (preprint).

10. Sibe Mardešić and Jack Segal, Shapes of compacta and ANR-systems, Fund. Math. 72 (1971), 41-59.

11. Leonard R. Rubin and Thomas J. Sanders, A shape theory for Hausdorff spaces (preprint).

Department of Mathematics, University of Georgia, Athens, Georgia 30602 